\title{
DETERMINACIÓN DEL EFECTO DEL CONSUMO DE LA TORTA DE SACHA INCHI (Plukenetia volúbilis L.) SOBRE EL PERFIL BIOQUÍMICO SANGUÍNEO DE POLLOS DE CARNE
}

\author{
Reiner REÁTEGUI-INGA,Daniel PAREDES-LÓPEZ², Rizal ROBLES-HUAYNATE² \\ 1 Practicante del Departamento de Ciencias Pecuarias, Facultad de Zootecnia, Universidad Nacional Agraria de la Selva. \\ Av. Universitaria s/n, PO BOX 156, Tingo María, Perú. \\ 2 Departamento de Ciencias Pecuarias, Facultad de Zootecnia, Universidad Nacional Agraria de la Selva. Av. Universitaria \\ s/n, PO BOX 156, Tingo María, Perú. E-mail: daniel.paredes9@gmail.com
}

\section{RESUMEN}

El objetivo de este estudio fue determinar los efectos de la torta de sacha inchi (TSI) en diferentes niveles de la ración de pollos sobre el perfil bioquímico sanguíneo, consumo de alimento (CAL), conversión alimenticia (CA) y ganancia de peso (GP), peso relativo de órganos e histología del hígado. Para esto se utilizaron 150 pollos ( 75 hembras y 75 machos) de 2 días de edad, de la línea Cobb 500, los cuales fueron divididos en 3 grupos o tratamientos (T1, T2 y T3). El T1 fue alimentado con $0 \%$, el T2 con $7 \%$ y el T3 con $14 \%$ de TSI. Las muestras de sangre fueron obtenidas a los 2, 12, 21 y 48 días de edad; el diseño estadístico usado fue el DCA con arreglo factorial $3 \times 3+1$. Los perfiles bioquímicos sanguíneos bajo efecto de la TSI fueron: $27.38 \pm 0.38,30.00 \pm 0.38$ y $29.56 \pm 0.45$ de hematocrito (\%); $2.86 \pm 0.10,104.51 \pm 2.44,92.74 \pm 1.96$ y $92.53 \pm 2.29$ de AST (UI/L); 30.81 $\pm 3.32,17.82 \pm 0.66$ y $20.95 \pm 0.62$ de ALT (UI/L) para T1, T2 y T3 respectivamente. Los niveles de TSI en la dieta no mostraron efecto $(\mathrm{p}>0.05)$ sobre los perfiles de hemoglobina, albúmina, proteína sérica, mientras que en los niveles de hematocrito, AST y ALT si tuvo efecto $(\mathrm{p}<0.05)$; pero los resultados mencionados se encontraron dentro de sus parámetros normales. En cuanto a CAL, CA y GP los niveles de TSI mostraron efecto adverso ( $p<0.05)$. La TSI tuvo efecto hepatotóxico, mas no así sobre el peso relativo de los órganos estudiados $(\mathrm{p}<0.05)$.

PALABRAS CLAVE: Sacha inchi, Plukenetia volúbilis L, perfil bioquímico sanguíneo.

\section{DETERMINATION OF THE EFFECT OF SACHA INCHI (Plukenetia volúbilis L.) CAKE FEEDING ON BLOOD BIOCHEMICAL PROFILE OF BROILERS}

\begin{abstract}
The aim of this study was to determine the effect of sacha inchi cake (SIC) in different levels of the diet of broiler chickens on the serum biochemical profile, feed intake (FI), feed conversion (FC), weight gain (WG), internal organs relative weight and liver histology. For this purpose, we used $150 \mathrm{Cobb}-500$ 2-day-old broilers chicken ( 75 males and 75 females) which were divided in 3 treatments (T1, T2 and T3). T1 was fed $0 \%, \mathrm{~T} 2,7 \%$ and T3, 14\% of SIC. Blood was sampled from yugular vein at 2 days old and wing vein at 12, 21 and 48 days old; a complete randomized design with a factorial $3 \times 3+1$ was used. The serum biochemical profiles under the PCSIC effect were: $27.38 \pm 0.38,30.00 \pm 0.38$ y $29.56 \pm 0.45$ for hematocrit; $104.51 \pm 2.44$, $92.74 \pm 1.96$ y $92.53 \pm 2.29$ for AST; $30.81 \pm 3.32,17.82 \pm 0.66$ and $20.95 \pm 0.62$ for ALT for T1, T2 y T3 respectively. Levels of SIC did not show effect $(\mathrm{p}>0.05)$ on hemoglobin, total protein, albumin, meanwhile, while these showed effect $(\mathrm{p}<0.05)$ on hematocrit, AST and ALT, but these results were between normal levels. SIC had negative effect on FI, FC and WG in T2 and T3 ( $>0.05)$ but did not had influence on the studied relative weight of internal organs $(\mathrm{p}>0.05)$. Liver tissue showed polynunleated hepatocytes in $\mathrm{T} 2$ and T3. In conclusion, SIC changed the hematocrit, AST and ALT levels and had hepatotoxic effect in liver tissue, feed intake, feed conversion and weight gain of broiler chicken when the level of SIC feeding increases.
\end{abstract}

KEYWORDS: Sacha inchi, Plukenetia volúbilis L, biochemical profile. 


\section{INTRODUCCIÓN}

Actualmente la avicultura se encuentra centralizada en la costa y en el norte del país, debido a esto, el abastecimiento de carne y huevos en nuestra Provincia de Leoncio Prado proviene de estas dos regiones. Sin embargo, nuestra provincia cuenta con excelentes condiciones climatológicas y ambientales, con insumos alimenticios regionales que podrían ser utilizados en la alimentación de aves, como la torta de sacha inchi, obtenida como residuo después de la extracción de aceite de la semilla, caracterizada por su alto valor proteico (59.13\%) (Brioso, 2007). Así, la torta de sacha inchi surge como una alternativa para sustituir los insumos proteicos, disminuyendo los costos de producción y consiguiendo que la oferta de productos sea mucho más adecuada.

La torta de sacha inchi procesada térmicamente se considera un insumo de alta calidad, ya que posee un alto nivel de proteína y energía bruta. Sin embargo, las semillas de sacha inchi contiene factores antinutricionales tóxicos que limitan el nivel de uso en especies monogástricas (Mondragón, 2009; Castillo et al., 2010). Estos factores podrían ser solucionados mediante el tratamiento térmico para mejorar la calidad del producto e incrementar su nivel de uso.

Los niveles de glucósidos y saponinas presentes en la torta de sacha inchi, después del proceso de cocción, causarían cambios en la estructura histológica del hígado y variación de los niveles de hemoglobina, hematocrito, proteína sérica, albúmina y transaminasa en sangre de pollos parrilleros. En ese contexto se genera la presente investigación, bajo el objetivo de determinar los efectos del consumo de la torta de sacha inchi en pollos de carne de la línea Cobb Vantres 500, sobre los perfiles de transaminasa, proteína sérica, hemoglobina, hematocrito, albúmina y desempeño zootécnico.

\section{MATERIALES Y MÉTODOS}

\section{Área de investigación y animales analizados}

El presente trabajo de investigación se ejecutó en las instalaciones de la Unidad Experimental de Aves y en el Laboratorio de Sanidad Animal de la Facultad de Zootecnia de la Universidad Nacional Agraria de la Selva, en Tingo María. Se usaron 150 pollos (75 hembras y 75 machos) de 2 días de edad, de la línea Cobb 500. Las aves fueron criadas bajo las mismas condiciones de alimentación, manejo y control sanitario.

\section{Preparación de las raciones}

Las raciones se formularon tomando como referencia los requerimientos nutricionales para aves propuestos por Cobb-vantress. (2008). El valor nutricional de las raciones usadas fueron de $21 \%$ de proteína total para los pollos entre 1 y 21 días de edad y $19 \%$ para los pollos entre 22 y 48 días. Así mismo, el contenido de energía fue de $2.988 \mathrm{kcal} / \mathrm{Kg}$ para la fase 1-12 días y de $3.000 \mathrm{Kcal} / \mathrm{Kg}$ para la fase de 13 48 días de edad.

\section{Recolección y procesamiento de muestras}

Los animales fueron muestreados a los 2, 12, 21 y 48 días de vida a tempranas horas y estando como mínimo una hora sin alimento. La extracción de sangre se realizó en la yugular para el día 2 y en la vena alar el resto de las extracciones. El hematocrito se determinó mediante microhematocrito en una centrífuga Hettich EBA 20, y la hemoglobina se determinó por el método de cianometahemoglobina. La alanina aminotransferas (ALT), aspartato aminotransferasa (AST), albúmina y proteína sérica se determinaron mediante reactivos de Wiener Lab y para las lecturas se usó un espectrofotómetro Boeco Germany S-22 UV/visible.

El control de peso de órganos se realizó a los 48 días de edad, una vez sacrificadas las aves. Los órganos pesados fueron hígado, páncreas, bursa, pulmón y bazo. Se usó una balanza electrónica con precisión decimal $(0.1 \mathrm{~g})$. Se usaron 2 pollos por repetición, los cuales contaban con pesos intermedios dentro de un mismo corral. Para la evaluación histológica del hígado, se utilizaron 2 aves que obtuvieron los pesos medios dentro de cada corral (repetición), estas fueron sacrificadas a los 48 días de edad, extrayéndoles muestras de hígado y fijándolas en solución de formol al 10\%.

Los parámetros zootécnicos fueron evaluados por periodos de 2 a 12 días de edad, 2 a 21 días de edad y 2 a 48 días de edad. Para el perfil bioquímico sanguíneo se usó el diseño completamente al azar (DCA) con arreglo factorial $3 \times 3+1$ (Tres niveles de TSIP y 3 tiempos + 1 tiempo, 2días); los datos fueron analizados mediante el análisis de variancia. Para evaluar los parámetros zootécnicos y el control de peso de los órganos se utilizó el DCA simple. Para el cálculo de las diferencias significativas mínimas entre las medias del tratamiento se utilizó el test de Student-Newman-Keuls (SNK). 


\section{RESULTADOS Y DISCUSIONES}

\section{Efecto del consumo de la torta de sacha inchi sobre el perfil bioquímico sanguíneo de pollos de engorde}

En el perfil bioquímico sanguíneo de pollos de engorde (Tabla 1), bajo el efecto de la TSIP no mostraron efecto sobre los perfiles de hemoglobina, albúmina, proteína sérica de los pollos, mientras que en los niveles de hematocrito, AST y ALT si tuvo efecto; en cuanto al efecto de la edad, los perfiles evaluados resultaron ser estadísticamente $(\mathrm{p}<0.05)$, pero los resultados mencionados se encontraron dentro de sus parámetros normales para ambos casos.

Los niveles de hemoglobina de pollos bajo los efectos de los niveles de TSIP, son estadísticamente iguales $(p>0,05)$. La igualdad puede deberse a la saponina posiblemente presente en la TSIP, la cual tiene acción hemolítica. No obstante, por vía oral este efecto es mínimo, debido a su bajo nivel de absorción por el tubo digestivo. Por otra parte Mondragón (2009); menciona que la baja concentración de ácido tánico en la torta de sacha inchi no perjudica su interacción con las proteínas u otros nutrientes.

Con respecto a la edad de las aves, la hemoglobina se vio afectada, siendo estos estadísticamente diferentes $(\mathrm{p}<0.05): \mathrm{La}$ hemoglobina bajó de $9.14 \mathrm{~g} / \mathrm{dL}$ a los 2 días hasta $7.89 \mathrm{~g} / \mathrm{dL}$ a los 12 días, para luego aumentar a $8.07 \mathrm{~g} / \mathrm{dL}$ y $11.2 \mathrm{~g} / \mathrm{dL}$ a los 21 y 48 días de vida respectivamente. Esta disminución inicial en las concentraciones de hemoglobina podría explicarse por un posible desbalance entre la síntesis de nuevos eritrocitos y la absorción de hierro, así como otras sustancias básicas en la formación de nueva hemoglobina, por lo que las cantidades sintetizadas de esta proteína, hasta ese momento, tendrían que redistribuirse en el total de nuevos eritrocitos y reticulocitos y esto se reflejaría en el descenso de las concentraciones de la proteína (Vásquez, 2011).

Los niveles de hematocrito de pollos bajo los efectos de los niveles de TSIP, son diferentes estadísticamente $(\mathrm{p}<0.05)$, siendo el T1 el que obtuvo menor valor comparado al T2 y T3, siendo estos últimos iguales entre sí. La diferencia tal vez se deba a que las aves del T2 y T3 desarrollaron una compensación de oxigenación para incrementar el desarrollo de tejidos por efecto de los glucósidos u otros FAN contenidos en la TSI, como es en el caso del hígado.

Con respecto a la edad de las aves, los niveles de hematocrito resultaron ser estadísticamente diferentes $(p<0.05)$. El hematocrito aumenta de acuerdo a la edad, siendo los niveles más altos y estadísticamente iguales entre sí a los 21 y 48 días de edad. Como indica Mateo (2006), los valores de hematocrito dependen de la edad y del sexo, siendo más elevados en edades adultas y/o en machos.

Los niveles de proteína sérica y albúmina bajo los efectos de los niveles de TSI, resultaron ser estadísticamente iguales $(p>0,05)$. La igualdad puede deberse a que las concentraciones de proteína y albúmina por lo general son normales en enfermedades hepáticas crónicas, pero en presencia de cirrosis o daño hepático los niveles de los mismos desciende notablemente (Ferato, 2010). En un ensayo en el cual se probaron dietas con diferentes niveles de inclusión $(0 \%, 5 \%, 10 \%$ y $15 \%)$ de frijol bayo (Vigna unguiculata L.) en pollos de engorde durante la fase de crecimiento, no se encontraron diferencias estadísticas $(\mathrm{p}<0.01)$ en la concentración de albúmina y proteína sérica (Miranda, et al., 2007).
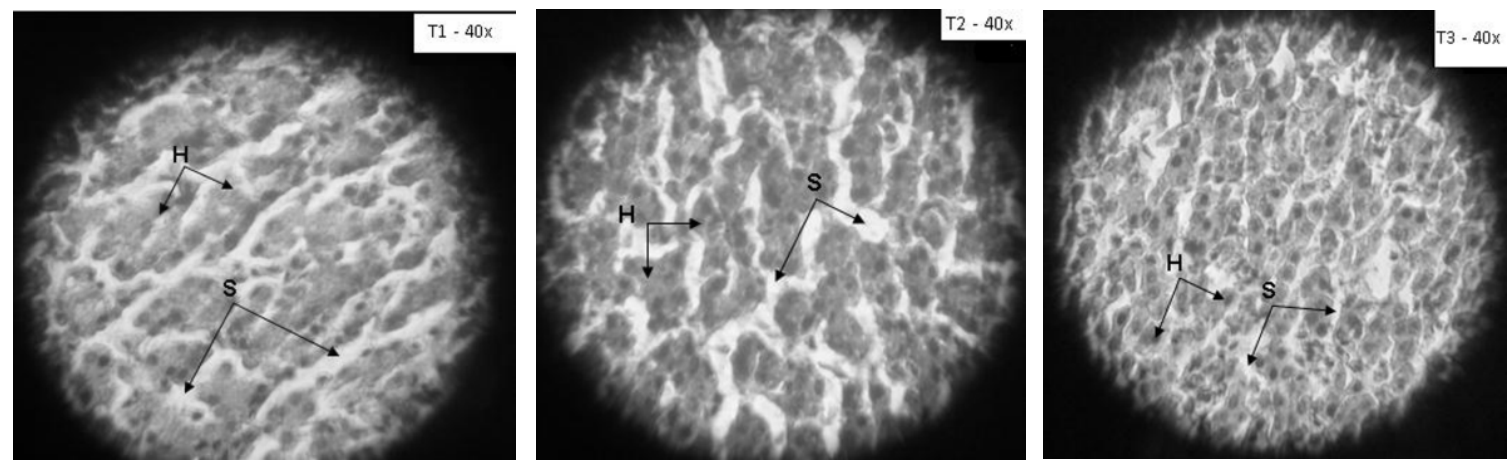

Figura 1. Fotografías del tejido hepático según la inclusión de TSI. H: Hepatocito, S: Sinusoide hepático 
Tabla 1. Efecto del consumo de TSIP y edad sobre el perfil bioquímico sanguíneo de pollos de carne de la línea cobb 500 en la etapa de levante.

\begin{tabular}{ccccccc}
\hline $\begin{array}{c}\text { Factores } \\
\text { evaluadas }\end{array}$ & $\begin{array}{c}\text { Hemoglobina } \\
(\mathbf{g} / \mathbf{d L})\end{array}$ & $\begin{array}{c}\text { Hematocrito } \\
(\%)\end{array}$ & $\begin{array}{c}\text { Proteína } \\
\text { sérica }(\mathbf{g} / \mathbf{d L})\end{array}$ & $\begin{array}{c}\text { Albúmina } \\
(\mathbf{g} / \mathbf{d L})\end{array}$ & $\begin{array}{c}\text { AST } \\
(\mathbf{U I} / \mathbf{L})\end{array}$ & $\begin{array}{c}\text { ALT } \\
(\mathbf{U I} / \mathbf{L})\end{array}$ \\
\hline Niveles de TSIP $(\%)$ & & & & & & \\
\hline 0 & $9.03 \pm 0.20 \mathrm{~A}$ & $27.38 \pm 0.38 \mathrm{~B}$ & $2.86 \pm 0.10 \mathrm{~A}$ & $1.11 \pm 0.02 \mathrm{~A}$ & $104.51 \pm 2.44 \mathrm{~A}$ & $30.81 \pm 3.32 \mathrm{~A}$ \\
\hline 7 & $9.17 \pm 0.23 \mathrm{~A}$ & $30.00 \pm 0.38 \mathrm{~A}$ & $2.98 \pm 0.11 \mathrm{~A}$ & $1.12 \pm 0.03 \mathrm{~A}$ & $92.74 \pm 1.96 \mathrm{~B}$ & $17.82 \pm 0.66 \mathrm{C}$ \\
14 & $8.99 \pm 0.24 \mathrm{~A}$ & $29.56 \pm 0.45 \mathrm{~A}$ & $2.82 \pm 0.10 \mathrm{~A}$ & $1.12 \pm 0.03 \mathrm{~A}$ & $92.53 \pm 2.29 \mathrm{~B}$ & $20.95 \pm 0.62 \mathrm{~B}$ \\
\hline Edad (días) & & & & & & \\
\hline 2 & $9.14 \pm 0.35 \mathrm{~B}$ & $24.93 \pm 0.98 \mathrm{C}$ & $2.25 \pm 0.14 \mathrm{C}$ & $0.96 \pm 0.05 \mathrm{C}$ & $134.73 \pm 0.64 \mathrm{~A}$ & $87.14 \pm 1.39 \mathrm{~A}$ \\
12 & $7.89 \pm 0.11 \mathrm{C}$ & $27.87 \pm 0.34 \mathrm{~B}$ & $2.90 \pm 0.06 \mathrm{~B}$ & $0.93 \pm 0.02 \mathrm{C}$ & $104.62 \pm 2.03 \mathrm{~B}$ & $15.63 \pm 0.55 \mathrm{D}$ \\
21 & $8.07 \pm 0.15 \mathrm{C}$ & $29.92 \pm 0.45 \mathrm{~A}$ & $2.06 \pm 0.04 \mathrm{C}$ & $1.13 \pm 0.02 \mathrm{~B}$ & $84.85 \pm 1.43 \mathrm{D}$ & $17.60 \pm 0.58 \mathrm{C}$ \\
48 & $11.20 \pm 0.13 \mathrm{~A}$ & $29.77 \pm 0.37 \mathrm{~A}$ & $3.86 \pm 0.06 \mathrm{~A}$ & $1.30 \pm 0.02 \mathrm{~A}$ & $92.76 \pm 2.05 \mathrm{C}$ & $22.27 \pm 0.52 \mathrm{~B}$ \\
\hline
\end{tabular}

No se encontró interacción entre TSIP y Edad, para todos los análisis realizados.

Con respecto a la edad de las aves, los niveles de proteína sérica obtenidos son estadísticamente diferentes $(\mathrm{p}<0.05)$, observándose que a los 2 días es igual a los 21 días de edad y estos menores a los 12 días de vida. El mayor valor encontrado fue a los 48 días, comportamiento similar obtenido por Jinez et al. (1998) en un ensayo de alimentación de pollos con semilla de jamaica (Hibiscus sabdariffa), encontrando a la cuarta semana $2.6 \mathrm{~g} / \mathrm{dL}$, mientras que a la séptima semana de vida encontró $5.34 \mathrm{~g} / \mathrm{dL}$ de proteína sérica.

Con respecto a la edad de las aves, los niveles de albúmina obtenidos en diferentes periodos de vida son estadísticamente diferentes $(\mathrm{p}<0.05)$, observándose un mayor valor a los 48 días comparado a los 2, 12 y 21 días de vida. En cuanto a los días 2 y 12 se observaron concentraciones de albúmina por debajo de lo normal. Por su parte Miranda et al. (2007), alimento a pollos con harina de granos de fríjol (Vigna unguiculata L. Walp) encontrando en el tratamiento control 1.66, $1.92 \mathrm{y}$ $1.61 \mathrm{mg} / \mathrm{dL}$ a la $1 \mathrm{ra}, 2 \mathrm{da}$ y $3 \mathrm{ra}$ semana de vida, no habiendo diferencia estadística $(\mathrm{p}<0.05)$.

Los niveles de aspartato aminotransferasa, bajo el efecto de los niveles de TSI de los pollos en estudio, son estadísticamente diferentes $(p<0.05)$. En el T1, se observa que la concentración de AST es mayor que los T2 y T3, probablemente debido al incremento de la mitosis de los hepatocitos (hiperplasia), ocasionado por glucósidos u otros FAN de la TSIP. Jínez et al. (1998), concluyó que no hubo ningún efecto de la semilla de Jamaica (Hibicus sabdariffa) sobre la concentración de AST, basándose en que lo datos registrados cayeron dentro de los parámetros normales (70 -220UI/L). Con respecto a la edad del ave, los niveles de aspartato aminotransferasa en diferentes periodos de vida, existe diferencia estadística ( $\mathrm{p}<0.05)$, pero estos se encuentran dentro de los parámetros normales.

Los niveles de ALT bajo los efectos de los niveles de TSI, resultaron ser estadísticamente diferentes $(p<0.05)$. Una disminución en la concentración de la ALT, tal como fue observado en las aves del tratamiento 2 y 3 , podría deberse también al incremento de tejido causado por los diferentes factores antinutricionales que posee la TSI, los cuales afectan el hígado, tratando de adaptarse y sobreponerse frente al estímulo. Por su parte, Jinez et al. (1998), realizó un estudio sobre el efecto de niveles elevados $(0 \%, 10 \%, 20 \%$ y $30 \%)$ de semilla de Jamaica (Hibicus sabdariffa) en dietas para pollos sobre el comportamiento productivo y funcionamiento hepático. Concluye que no hubo ningún efecto de la semilla de Jamaica sobre la concentración de ALT, basándose en que lo datos registrados cayeron dentro de los parámetros normales.

Por otro lado, en un ensayo en el cual se administró a un grupo de ratones machos de la cepa Balb C57, por vía oral, $0.5 \mathrm{ml}$ de aceite de sacha inchi por $\mathrm{kg}$ de peso vivo (grupo problema) y a otro grupo (control) $4 \mathrm{ml}$ de suero fisiológico por $\mathrm{kg}$ de peso vivo, ambos de 3 hasta 60 días de vida, se encontró a los 30 días $16 \pm 3.6$ y $14.8 \pm 3.5 \mathrm{UI} / \mathrm{dL}$ de ALT en el grupo problema y control respectivamente y a los 60 días $19 \pm 1.2 \mathrm{UI} / \mathrm{dL}$ y $16.9 \pm 8.3 \mathrm{UI} / \mathrm{Dl} \mathrm{de}$ ALT en el grupo problema y control respectivamente. En ambas evaluaciones $(30$ y 60 días) no se encontró diferencia estadística $(\mathrm{P}>0,05)$ (Gorriti et al, 2010). 
Con respecto a la edad de las aves, los niveles de ALT obtenidos en diferentes etapas de vida resultaron ser estadísticamente diferentes $(\mathrm{p}<0.05)$; observándose una disminución progresiva a los 2,12 y 21 días de edad para luego aumentar a los 48 días; observando en la primera evaluación la concentración de ALT más elevada, siendo probablemente debido a un estrés fisiológico de adaptación normal. En cuanto a los 12, 21 y 48 días se observa un incremento progresivo de ALT; en un ensayo realizado por Gorrirti et al, (2010); encontró el mismo comportamiento; en ratones sanos machos de la cepa Balb C57 (ratones de laboratorio); encontró en promedio $16 \pm 3.6 \mathrm{UI} / \mathrm{dL}$ y $19 \pm 12 \mathrm{UI} / \mathrm{dL}$ deALT a los 30 y 60 días de vida respectivamente.

\section{Efecto del consumo de la torta de sacha inchi sobre el tejido hepático}

La tabla 2 muestra la descripción de la histología hepática de pollos a los 48 días de edad, alimentados con torta de sacha inchi, observándose que el T3 presenta una mayor concentración de hepatocitos, debido a que una de las causas más comunes de hiperplasia es la irritación crónica por presencia de toxinas, lo cual provoca la proliferación y el acumulo de las células (Cheville, 1996). Uno de los componentes que pudo causar la hiperplasia hepática son los glucósidos; como indican Mondragón (2009); Castilo, et al.,, (2010), la presencia de glucósidos en la torta de sacha inchi es abundante $(+++)$; por su parte González (2010) indica que los glucosinolatos al ser hidrolizados generan glucosa, ácido sulfúrico y compuestos volátiles como isotiocianatos, oxazolidin-2-tionas, tiocianatos y nitrilos; causando estos últimos lesiones en el hígado.

De manera general, se puede deducir que en la TSI existen factores antinutricionales o sustancias nocivas, ya que la histología hepática del T2 y T3 resultó afectada en comparación del T1. Por su parte, Cornell university (2009), indica que los síntomas de intoxicación de glucósidos en aves de corral son varias, entre ellas la lesión hepática.

\section{Efecto del consumo de la torta de sacha inchi sobre el peso relativo de los órganos de pollos de engorde}

En la Tabla 3 se muestra el peso promedio relativo de diferentes órganos de pollos de 48 días de edad alimentados con diferentes niveles de torta de sacha inchi. El peso relativo de los órganos evaluados son estadísticamente iguales $(\mathrm{p}>0,05)$. Arrieta et al. (2006), demostró que los hígados del tratamiento con $0,07 \mathrm{mg}$ de aflatoxina/ $\mathrm{kg}$ de alimento balanceado (T2) no variaron significativamente su peso relativo con respecto al control (T1) y no presentaron lesiones macroscópicas, adjudicándole estos resultados a las bajas concentraciones de aflotoxina ingerida en la dieta. Sin embargo, los pollos que recibieron dietas con niveles de aflatoxina $(0,075 \mathrm{mg} / \mathrm{kg})$ similares a los de este estudio durante 42 días, demostraron un significativo incremento de los lípidos del hígado y consecuentemente del tamaño del mismo.

Por su parte, Santin et al. (2003), indica que el peso relativo promedio de la bursa de Fabricio es de $0.17 \%$ a los 42 días de vida. Asimismo, Tambini et al., (2010); en un ensayo, encontró $0.167 \%$ y $0.174 \%$ de peso relativo de bursa y bazo respectivamente en pollos clínicamente sanos de 49 días de vida; siendo estos casi similares a los encontrados en el presente estudio.

\section{Efecto del consumo de la torta de sacha inchi sobre desempeño zootécnico de los pollos}

Los resultados obtenidos se muestran en la tabla 4 , donde se observa las variables de consumo diario de alimento (CDA, g), ganancia diaria de peso (GDP, g) y conversión alimenticia (CA) de pollos alimentados con diferentes niveles de inclusión de torta de sacha inchi. En los tres periodos de evaluación se observaron diferencias significativas

Tabla 2. Descripción del tejido hepático de pollos a los 48 días de edad alimentados con torta de sacha inchi..

\begin{tabular}{cccc}
\hline Partes & T1 (0\% TSIP) & T2 (7\% TSIP) & T3 (14\% TSIP) \\
\hline $\begin{array}{c}\text { Sinusoides } \\
\text { hepáticos }\end{array}$ & $\begin{array}{c}\text { Arquitectura } \\
\text { normal }\end{array}$ & $\begin{array}{c}\text { Efectos no muy } \\
\text { marcados }\end{array}$ & $\begin{array}{c}\text { Luz sinusoidal } \\
\text { disminuida }\end{array}$ \\
Hepatocitos & $\begin{array}{c}\text { Arquitectura } \\
\text { normal }\end{array}$ & $\begin{array}{c}\text { Aparición de hepatocitos } \\
\text { polinucleados }\end{array}$ & $\begin{array}{c}\text { Aumento considerable de volumen } \\
\text { de los hepatocitos polinucleados }\end{array}$ \\
\hline
\end{tabular}


$(\mathrm{p}<0.05)$ para las variables evaluadas, con excepción de la conversión alimenticia en el periodo de 2 - 21 días de edad, donde no reportaron diferencia significativa $(\mathrm{p}>0.05)$ entre los tres tratamientos.

En el periodo de 2 - 12 días de edad, se observó que los pollos alimentados con raciones incluidas de $0 \%$ y $7 \%$ de TSI consumieron más alimento $(\mathrm{p}<0.05)$ en relación a los pollos alimentados con $14 \%$ de TSI. Entre tanto, para la ganancia de peso se observó que a mayor inclusión de TSIP en ración de pollos, menor ganancia de peso $(\mathrm{p}<0.05)$. La conversión alimenticia de pollos alimentados con $7 \%$ fueron semejantes $(\mathrm{p}>0.05)$ a los animales del $\mathrm{T} 1$ $(0 \%$ TS) y T3 (14\% TSI); entre tanto, el grupo de animales que consumieron $14 \%$ de TSI tuvieron mayor conversión alimenticia en relación a los pollos alimentados con $0 \%$ de TSIP.

El pobre desempeño zootécnico hallado en las aves del T3 podría deberse a la mayor cantidad de glucósidos y saponinas presentes en su alimento, ya que los compuestos derivados de los glucósidos son los isotiocianatos, oxazolidina-2-tionas y nitrilos, y estos tienen la capacidad de suprimir el crecimiento (Cornell University, 2009). Las saponinas interfieren en la absorción intestinal y causan trastornos metabólicos, ya que se unen a nutrientes como al zinc inhibiendo así la producción de enzimas metabólicas y digestivas (Mondragón, 2009). Las saponinas y los glucósidos afectan la palatabilidad, ya que son amargos y picantes respectivamente (Gonzales et al., 2011). En el periodo de 2 - 21 días de edad se observa que los pollos alimentados con $0 \%$ y $7 \%$ de TSI tienen semejante $(\mathrm{p}<0.05)$ CAD y GPD y diferente $(\mathrm{p}<$ $0.05)$ a los pollos del T3 (14\% TSI). Lo que se puede deducir que las aves del T2 posiblemente trataban de adaptarse al alimento, mientras que las aves del T3 no lo consiguieron porque sus parámetros productivos sucumbieron, ya que su dieta contenía $14 \%$ de TSI y por ende mayor cantidad de sustancias antinutricionales. Mientras que el bajo consumo de alimento por parte de las aves, posiblemente se deba a que las saponinas afecta negativamente la palatabilidad y actúan sobre la mucosa intestinal reduciendo su capacidad de absorción (Mondragón, 2009; Boza, 1991).

En el periodo de 2 - 48 días de edad, observamos que una mayor inclusión de TSIP en las raciones de pollos, provoca pobre desempeño zootécnico, posiblemente debido a que la cantidad de consumo de alimento (TSI) es mayor. Por su parte Mondragón (2009), indica que la presencia de saponinas y glucósidos en la torta de sacha inchi es abundante $(+++)$. Los glucósidos al ser hidrolizados por una enzima hidrolítica originan glucosa y algunos derivados del aglucón como tiocianatos, nitrilos u oxazolidintionas y isotiocianatos. Estos últimos causan gastroenteritis, reduciendo así la absorción de los nutrientes y el retraso en el crecimiento (Parada, 2003). Los niveles elevados de saponinas originan problemas sensoriales relacionados con la mayor astringencia y amargor, causando el rechazo por parte del animal (Gonzales et al., 2011)

Por su parte, Reátegui et al. (2010), al incluir 0\%, $20 \%, 30 \%$ y $40 \%$ de torta de sacha inchi en raciones de pollos, encontró diferencia estadística $(\mathrm{p}<0.01)$ a los 45 días de edad, en el peso vivo, consumo de alimento y la conversión alimenticia, mostrando que a mayor inclusión de TSIP, se provoca una caída en la ganancia de peso, consumo de alimento y alto índice de conversión alimenticia. El rendimiento productivo recomendado para pollos de la línea Cobb 500 a los 49 días de edad, es de $123 \mathrm{~g}$ de CDA, $65 \mathrm{~g}$ de GDP y 1.9 de CA, siendo estos similares a los obtenidos en el T1 del presente estudio (CobbVantress , 2008);

Tabla 3. Peso promedio relativo ( $\mathrm{g} / 100 \mathrm{~g}$ de peso vivo) de órganos de pollos de 48 días de vida alimentados con diferentes niveles de torta de sacha Inchi precocida.

\begin{tabular}{|c|c|c|c|c|c|}
\hline \multirow{2}{*}{ Órganos } & \multicolumn{3}{|c|}{ Tratamientos } & \multirow{2}{*}{$\begin{array}{l}\text { CV } \\
(\%)\end{array}$} & \multirow{2}{*}{$\begin{array}{l}\text { Sig. }{ }^{1} \\
(0.05)\end{array}$} \\
\hline & $0 \%$ & $7 \%$ & $14 \%$ & & \\
\hline Hígado & $1.70 \mathrm{~A}$ & $1.66 \mathrm{~A}$ & $1.75 \mathrm{~A}$ & 7.94 & NS \\
\hline Páncreas & $0.17 \mathrm{~A}$ & $0.19 \mathrm{~A}$ & $0.18 \mathrm{~A}$ & 10.86 & NS \\
\hline Bursa & $0.14 \mathrm{~A}$ & $0.18 \mathrm{~A}$ & $0.17 \mathrm{~A}$ & 21.01 & NS \\
\hline Pulmón & $0.64 \mathrm{~A}$ & $0.58 \mathrm{~A}$ & $0.66 \mathrm{~A}$ & 13.17 & NS \\
\hline Baso & $0.10 \mathrm{~A}$ & $0.10 \mathrm{~A}$ & $0.10 \mathrm{~A}$ & 20.90 & NS \\
\hline
\end{tabular}

- NS: No significatico.

- CV $=$ Coeficiente de variación 
Tabla 4. Promedios \pm error estándar del CAL, GP en gramos/día y CA de pollos alimentados con diferentes niveles de TSIP en función a los periodos evaluados.

\begin{tabular}{|c|c|c|c|c|c|}
\hline Parámetros ${ }^{1}$ & T1 & $\mathrm{T} 2$ & T3 & C.V. & $\mathbf{p}$ \\
\hline \multicolumn{6}{|c|}{ Periodo: 2 - 12 días de edad. } \\
\hline CAL & $38.00 \pm 0.30 \mathrm{~A}$ & $36.00 \pm 0.48 \mathrm{~A}$ & $33.00 \pm 1.00 \mathrm{~B}$ & 4.18 & $\mathrm{p}<0.05$ \\
\hline GP & $28.00 \pm 0.52 \mathrm{~A}$ & $25.00 \pm 0.72 B$ & $22.00 \pm 0.58 \mathrm{C}$ & 5.50 & $p<0.05$ \\
\hline $\mathrm{CA}$ & $1.35 \pm 0.03 \mathrm{~A}$ & $1.42 \pm 0.29 \mathrm{AB}$ & $1.51 \pm 0.04 \mathrm{~B}$ & 5.00 & $\mathrm{p}<0.05$ \\
\hline \multicolumn{6}{|c|}{ Periodo: 2 - 21 días de edad. } \\
\hline CAL & $69.00 \pm 0.72 \mathrm{~A}$ & $68.00 \pm 1.15 \mathrm{~A}$ & $59.00 \pm 1.65 B$ & 4.24 & $\mathrm{p}<0.05$ \\
\hline GP & $44.00 \pm 0.65 \mathrm{~A}$ & $42.00 \pm 0.54 \mathrm{~A}$ & $36.00 \pm 0.79 B$ & 3.66 & $p<0.05$ \\
\hline CA & $1.56 \pm 0.01$ & $1.63 \pm 0.02$ & $1.62 \pm 0.05$ & 4.39 & $\mathrm{p}<0.05$ \\
\hline \multicolumn{6}{|c|}{ Periodo: 2 - 48 días de edad. } \\
\hline CAL & $124.00 \pm 0.46 \mathrm{~A}$ & $102 \pm 0.86 B$ & $88.00 \pm 0.50 \mathrm{C}$ & 1.35 & $p<0.05$ \\
\hline GP & $64.00 \pm 0.40 \mathrm{~A}$ & $46.00 \pm 1.25 B$ & $34.00 \pm 0.70 \mathrm{C}$ & 4.04 & $p<0.05$ \\
\hline CA & $1.94 \pm 0.02 \mathrm{~A}$ & $2.26 \pm 0.05 B$ & $2.60 \pm 0.06 \mathrm{C}$ & 4.47 & $\mathrm{p}<0.05$ \\
\hline
\end{tabular}

Letras diferentes dentro de la columna para cada periodo, indican diferencia estadística (NSK 5\%)

\section{CONCLUSIÓN}

Los niveles de TSIP no mostraron efecto sobre los perfiles de hemoglobina, albúmina, proteína sérica de los pollos. Los niveles de hematocrito, AST y ALT si se vieron afectados. Se observó un efecto adverso sobre el consumo de alimento, conversión alimenticia y ganancia de peso, mas no hubo efecto sobre el peso relativo de los órganos estudiados. En el tejido hepático del T3 se observó la disminución del lumen de los espacios sinusoides y un incremento de núcleo en los hepatocitos a comparación del T1.

\section{AGRADECIMIENTOS}

Al Sr. Félix Jara Ramírez, Laboratorista del laboratorio de Sanidad Animal de la Facultad de Zootecnia, por su apoyo en los análisis realizados. Al Ing. Hugo Saavedra Rodríguez, por su apoyo en el manejo de las aves.

\section{BIBLIOGRAFÍA CITADA}

Arrieta, D.; Pérez M.; Gómez C.; Molero G.; Novoa E.; Rincón H.; Ascanio E. 2006. Efecto Del Alimento Contaminado Con Aflatoxina B1 $(0,07$ $\mathrm{Mg} / \mathrm{Kg}$ ) Sobre La Morfología Hepática Y Actividad Enzimática Sérica (Ast Y Alt) En Pollos De Engorde. (http://www.saber.ula.ve/ bitstream/123456789/28388/2/art5.pdf) Acceso: 11/11/2012.
Brioso, B. 2007. Evaluación del valor nutricional y energía metabolizable del sacha inchi (Plukenetia volubilis L Walp) integral en pollos de carne. Tesis de pre-grado. Universidad Nacional Agraria de la Selva, Tingo María. 45pp

Boza, J. SA. Valor nutritivo de las leguminosas grano en la alimentación humana y animal. (http://www.insacan.org/racvao/anales/1991/ articulos/03-1991-07.pdf) Acceso: 19/05/2013.

Castillo, E; Castillo, S; Reyes, E. 2010. Estudio fitoquímico de Plukenetia volubilis L. y su efecto antioxidante en la lipoperoxidación inducida por $\mathrm{Fe} 3+/$ ascorbato en hígado de Rattus rattus var. A l binus. Revistas. Concytec (http://revistas.concytec.gob.pe/pdf/scientia/v2 n1/a02v2n1.pdf)Acceso: 19/05/2013).

Cheville, N. 1994. Ultrastructural Pathology: The comparative cellular basis of diseases. Iowa State University Press. IA. 946pp.

Cobb-Vantress. 2008. Suplemento informativo de rendimiento y nutrición de pollo de engorde. (http://www.cobb-vantress.com/contactus/ brochures/Cobb700_BPN_SupplementSpanish. pdf). Acceso: 3/04/2013)

Cornell University, 2009. Plants Poisonous to Livestock: Glucosinolates (Goitrogenic Glycosides). (http://www.ansci.cornell.edu/ plants/toxicagents/glucosin.html). Acceso: $1 / 06 / 2013$ 
Ferato. 2010 . A 1 bú min Ferato (http://www.ferato.com/wiki/index.php/Alb\%C 3\%BAmina). Acceso: 15/09/2012).

Gonzáles J. 2010. Biomoléculas: saponinas: EHU (http://www.ehu.es/biomoleculas/hc/sugar33c1. htm, Acceso: 15 de septiembre del 2012).

Gonzales, G; Pérez, M; Frikha, M; Lázaro, R; y García, D. 2011. Composición nutricional del guisante. (http://albeitar.portalveterinaria.com/ noticia/ $10173 /$ ART \%C 3\% 8DCULOS NUTRICI\%C3\%93N/composici\%C3\%B3nnutricional-guisante-ii.html), Acceso: 1/06/2013).

Gorriti, A.; Arroyo, J.; Quispe, F.; Cisneros, B.; Condorhuamán, M.; Almora, Y.; Chumpitaz V. 2010. Toxicidad oral a 60 días del aceite de sacha inchi (Plukenetia volubilis 1.) Y linaza (Linum usitatissimum 1.) y determinación de la dosis letal 50 en roedores. (www.scielo.org.pe/pdf/rins/ v27n3/a07v27n3.pdf). Acceso: 23/05/2012)

Jínez, T., Cortés, C., Ávila, E., Casaubon, T.; Salcedo, R. 1998. Efecto de niveles elevados de semilla de jamaica (Hibiscus sabdariffa) en dietas para pollos sobre el comportamiento productivo y funcionamiento hepático. (http://www.medigraphic.com/pdfs/vetmex/vm -1998/vm981f.pdf). Acceso: 30/03/2013).

Mateo, R. 2006. El valor hematocrito. (http://www.mailxmail.com/curso-analisisclinicos-rutina/valor-hematocrito, Acceso: 27 de abril del 2013)

Miranda, S.; Rincón, H.; muñoz, R.; Higuera, A.; Arzálluz, A.; Urdaneta, H. 2007. Parámetros productivos y química sanguínea en pollos de engorde alimentados con tres niveles diéticos de harina de granos de fríjol (Vigna unguiculata (l.) Walp.) Durante la fase de crecimiento. (http://redalyc.uaemex.mx/pdf/959/95917208.p df). Acceso: 15/05/2013.

Mondragón, G. 2009. Estudio farmacognóstico y bromatológico de los residuos industriales de la extracción del aceite de Plukenetia volubilis L. (Sacha inchi). Tesis de Pre-grado. Universidad Nacional Mayor de San Marcos, Lima. 127pp.
Reátegui, V.; Flores, J.; Ramírez, J.; Yalta, R.; Manrique, J.; D'azevedo, G.; Pinedo J.; Bardales, J.; Machuca, G.; Rengifo, O.; Rengifo, D.; D'azevedo, A. 2010. Evaluación de la Torta de Sacha Inchi (Plukenetia volubilis) y su uso como fuente alternativa y proteica en la alimentación de pollos de engorde y gallinas de postura en Zungaro Cocha - UNAP. Universidad Nacional De La Amazonia Peruana, Iquitos - Perú. 20pp.

Santin, E.; Paulillo, A.; Krabbe, E.; Alessi, A.; Polveiro, W.; Maiorka, A. 2003. Low level of aflatoxin in broiler at experimental conditions. use of cell wall yeast as adsorbent of aflatoxin. (journals.ut.ac.ir/page/download1qNirlLrXY.artdl). Acceso: 3/04/2012).

Tambini, A.; Alba, M.; Perales, R.; Falcón, N. 2010. Evaluación anátomo-histopatológica de bursa, timo y Bazo de pollos de carne criados sobre cama reutilizada vs. Cama nueva. (http://www.scielo.org.pe/pdf/rivep/v21n2/a06v 21n2.pdf). Acceso: 5/06/2013).

Universidad de Zaragoza. 2011. Valores h e m a tológ i cos normales. (http://cea.unizar.es/Disenos_experimentales/ Sangre/VALORES\%20HEMATOLOGICOS.pd f). Acceso: 15/05/2012).

Vásquez, L. 2011. Efecto de la exposición a 2638 msnm, desde el día 21 de edad, en los valores de hematocrito, concentración de hemoglobina y en el peso pulmonar de pollos de la estirpe comercial Cobb, según el sexo. http://www.bdigital.unal.edu.co/5343/1/ isabelcristinavasquezvelez.2011.pdf, Acceso: 27 de abril del 2013).

Recibido: 13 de agosto del 2015

Aceptado para publicación: 1 de octubre del 2015 